\title{
Benzocaína e eugenol como anestésicos para o quinguio (Carassius auratus)
}

\author{
[Benzocaine and eugenol as anesthetics for golden fish (Carassius auratus)] \\ F. Bittencourt ${ }^{1}$, B.E. Souza ${ }^{2}$, W.R. Boscolo ${ }^{1}$, R.R. Rorato ${ }^{3}$, A. Feiden ${ }^{1}$, D.H. Neu ${ }^{4}$ \\ ${ }^{1}$ Unioeste -Toledo, PR \\ ${ }^{2}$ IFPR - Foz do Iguaçu, PR \\ ${ }^{3}$ Unioeste - Toledo, PR (in memoriam) \\ ${ }^{4}$ Aluno de pós-graduação - Universidade Estadual de Maringá - UEM - Maringá, PR
}

\begin{abstract}
RESUMO
Avaliaram-se os tempos de indução e recuperação de quinguios (Carassius auratus) expostos a dois

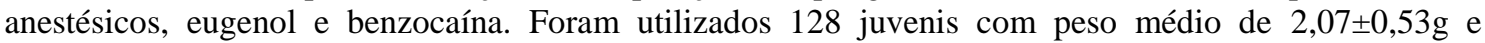
comprimento total médio de $5,51 \pm 0,56 \mathrm{~cm}$. A benzocaína mostrou ser mais eficiente do que o eugenol em relação ao tempo, tanto para indução ao coma quanto para a recuperação à fuga e também no que diz respeito à sobrevivência. As doses de benzocaína com melhores resultados foram de 87,5 e $100 \mathrm{mg} . \mathrm{L}^{-1}$. O eugenol proporcionou demora na indução e na recuperação dos animais, além de ter apresentado mortalidades quando as doses anestésicas foram elevadas.
\end{abstract}

Palavras-chave: peixe ornamental, anestesia, manejo

\begin{abstract}
The aim of this work was to evaluate the induction and recuperation time of quinguio (Carassius auratus) exposed to two anesthetics, eugenol and benzocaine. 128 juveniles with $2.07 \pm 0.53 \mathrm{~g}$ of average weight and $5.51 \pm 0.56 \mathrm{~cm}$ of total length were used. The benzocaine proved to be more efficient than the eugenol regarding the time in inducing a coma and recovering flight, as well as survival. The better results of benzocaine doses were 87.5 and $100 \mathrm{mg} . \mathrm{L}^{-1}$. The eugenol resulted in a delay of animal induction and recovery, and also presented mortalities when the anesthetics doses were increased.
\end{abstract}

Keywords: anesthesia, ornamental fish, management

\section{INTRODUÇÃO}

O gênero Carassius ocupa lugar de destaque na comercialização mundial de peixes ornamentais para aquariofilia (Soares et al., 2000). Em função da sua aparência, docilidade durante o manejo, rusticidade frente as condições ambientais e adaptação ao confinamento em locais pequenos (Bandyopadhyay et al., 2005), esse peixe tem grande cultivo por todo o mundo.

Como as espécies produzidas em aquários sofrem fortes perturbações durante seu manejo, é interessante a utilização de anestésicos para reduzir o estresse dos animais e evitar a morte

Recebido em 9 de março de 2011

Aceito em 28 de agosto de 2012

E-mail: bitanca@hotmail.com desses peixes. O uso dessas substâncias tem demonstrado eficácia para contornar eventuais transtornos nas práticas de manejo aplicadas no cotidiano da criação de peixes, podendo reduzir significativamente os aspectos negativos de uma produção piscícola.

A benzocaína é um dos principais anestésicos utilizados para a imobilização de peixes durante o manejo (Inoue et al., 2002). Além de ser o produto mais disponível no Brasil para tal prática (Gomes et al., 2001) e seu uso ser ecologicamente correto, pode ser utilizado frequentemente sem causar problemas reprodutivos ou diminuição do crescimento (Roubach e Gomes, 2001). 
O eugenol é um fármaco alternativo de origem vegetal (Honczaryk e Inoue, 2009). É o principal componente do óleo de cravo e, de acordo com Woody et al. (2002), é eliminado da corrente sanguínea em menos de dois dias após sua utilização. Entretanto, quando aplicadas doses elevadas, pode acarretar prejuízos para um cultivo devido à sua toxidade.

Segundo Roubach e Gomes (2001), para biometrias ou qualquer outro manuseio de peixes que necessite de anestesia profunda, para segurança dos animais, esse estágio deve ser atingido entre 1 a 3 minutos, com tempo de recuperação inferior a 5 minutos.

No Brasil, a resolução 714, de 2002, do Conselho Federal de Medicina Veterinária, autoriza a eutanásia de peixes com o uso de barbitúricos, anestésicos inaláveis, $\mathrm{CO}_{2}$, tricaína metano sulfonato (TMS, MS222), hidrocloreto de benzocaína e 2-fenoxietanol. Mesmo assim, ainda há preocupação com a aplicação dessas substâncias.

Como a manipulação dos peixes é imprescindível para o cultivo de animais em ambientes confinados, o objetivo deste estudo foi avaliar os tempos de indução e recuperação de quinguios (C. auratus) submetidos à insensibilização por dois anestésicos, benzocaína e eugenol.

\section{MATERIAL E MÉTODOS}

O trabalho foi realizado no Laboratório de Aquicultura da Universidade Estadual do Oeste do Paraná - Unioeste, Campus de Toledo, PR, em conjunto com o Grupo de Estudos de Manejo na Aquicultura - GEMAq. Foram utilizados 128 juvenis de quinguio (C. auratus) com peso médio de $2,07 \pm 0,53 \mathrm{~g}$ e comprimento total médio de $5,51 \pm 0,56 \mathrm{~cm}$, adquiridos em uma piscicultura comercial localizada na região oeste do estado do Paraná. Os animais foram aclimatados durante sete dias em uma caixa de $1.000 \mathrm{~L}$ com aeração e renovação de água constantes. A alimentação foi realizada até a saciedade aparente, quatro vezes ao dia, com ração comercial contendo $32 \%$ de proteína bruta processada na forma farelada. Diariamente realizaram-se duas sifonagens (manhã e tarde) para retirada de fezes e ração não consumida.

O experimento foi realizado em aquários de vidro com volume útil de $30 \mathrm{~L}$, mas contendo apenas $10 \mathrm{~L}$ de água, retirada do próprio tanque de aclimatação, com temperatura de $25^{\circ} \mathrm{C}$. Foram aferidos o oxigênio dissolvido, o $\mathrm{pH}$ e a condutividade elétrica. As soluções de eugenol bem como de benzocaína foram diluídas em álcool etílico $(98,8 \%)$, resultando em duas soluções estoque na proporção de $100 \mathrm{mg} \cdot \mathrm{mL}^{-1}$ (1:10).

Testaram-se as concentrações de $0 ; 12,5 ; 25$; 37,$5 ; 50 ; 62,5 ; 75 ; 87,5 ; 100$ e $112,5 \mathrm{mg}^{-L^{-1}}$ para benzocaína e 12,$5 ; 25 ; 37,5 ; 50 ; 62,5$; e $75 \mathrm{mg} \mathrm{L}^{-1}$ para o eugenol. Os testes com cada anestésico foram realizados individualmente e em dias separados, da menor concentração para a maior. Utilizaram-se 8 peixes $(n=8)$ expostos individualmente a cada tratamento. $\mathrm{O}$ controle recebeu o mesmo manejo que os demais, porém sem a solução anestésica no aquário.

O tempo necessário para o aparecimento dos padrões comportamentais avaliados foi monitorado por meio de cronômetro digital. A água dos aquários foi totalmente trocada a cada teste.

Os estágios anestésicos foram verificados de acordo com a Tab. 1 .

Tabela 1. Características comportamentais dos peixes de acordo com os diferentes estágios de anestesia*

\begin{tabular}{cl}
\hline Estágio & Característica de comportamento \\
\hline 1 & $\begin{array}{l}\text { Movimento opercular visivelmente lento ou errático } \\
\text { Perda parcial de equilíbrio e dificuldade de manter posição normal de nado, quando } \\
\text { parado }\end{array}$ \\
3 & $\begin{array}{l}\text { Perda total de equilíbrio e incapacidade de recuperar a posição vertical de nado } \\
\text { ("barriga para cima") }\end{array}$ \\
4 & $\begin{array}{l}\text { Ausência de reação a qualquer estímulo } \\
\text { Recuperação da posição normal de nado e da capacidade de nadar }\end{array}$ \\
\hline
\end{tabular}

*Adaptado de Woody et al. (2002) 
A ausência de reação a qualquer estímulo foi verificada pelo toque na lateral dos peixes com um bastão de vidro. A recuperação anestésica realizou-se individualmente, em aquário contendo 20L de água sem anestésico, com aeração constante. Após o restabelecimento dos animais, os organismos foram acondicionados em aquários de 30L, onde foram mantidos com aeração constante e alimentação durante 96 horas para monitoramento da mortalidade.

Utilizaram-se análises de variância (ANOVA Unifatorial), com posterior comparação múltipla de médias pelo teste Tukey, com 5\% de significância, no programa estatístico Statistical Analysis System (SAS, 2004).

\section{RESULTADOS E DISCUSSÃO}

Os parâmetros físicos e químicos da água, mensurados durante o experimento como $\mathrm{pH}$ $6,79 \pm 0,23$, condutividade elétrica $83,6 \pm 3,28$ $\mu \mathrm{S} . \mathrm{cm}^{-1}$ e oxigênio dissolvido $5,01 \pm 0,45 \mathrm{mg} . \mathrm{L}^{-1}$, permaneceram situados dentro dos limites confortáveis para carpas, conforme Golombieski et al., 2005.

Em todas as concentrações de benzocaína, exceto para as inferiores a $50 \mathrm{mg} . \mathrm{L}^{-1}$, os peixes atingiram o estágio de anestesia profunda (Tab. 2). A melhor dose encontrada foi de $87,5 \mathrm{mg} . \mathrm{L}^{-1}$, mesmo não sendo significativamente diferente no tempo de sedação quando dispostas dosagens maiores; entretanto, os intervalos de recuperação são mais acentuados quando utilizadas concentrações superiores. $\mathrm{O}$ tratamento de $50 \mathrm{mg} . \mathrm{L}^{-1}$ teve tempo de anestesia maior e tempo de recuperação longo quando comparado aos outros teores do fármaco.

Houve diferenças significativas $(\mathrm{P}<0,05)$ entre as concentrações tanto para a indução quanto para a recuperação. Para a sedação dos animais, houve efeito exponencial, quando se aumenta a dose, o tempo de indução diminui.

Tabela 2. Tempo (em segundos) de indução e recuperação dos quinguios expostos a diferentes concentrações de benzocaína

\begin{tabular}{lcc}
\hline Tratamento $\left(\mathrm{mg} . \mathrm{L}^{-1}\right)^{*}$ & Anestesiado & Recuperado \\
\hline 50,0 & $523,5 \pm 58,6 \mathrm{c}$ & $300,0 \pm 95,2 \mathrm{ab}$ \\
62,5 & $364,0 \pm 65,2 \mathrm{~b}$ & $262,7 \pm 44,0 \mathrm{ab}$ \\
75,0 & $324,4 \pm 28,8 \mathrm{~b}$ & $235,1 \pm 29,3 \mathrm{a}$ \\
87,5 & $239,0 \pm 43,2 \mathrm{a}$ & $250,8 \pm 60,0 \mathrm{a}$ \\
100,0 & $231,7 \pm 54,2 \mathrm{a}$ & $347,7 \pm 55,6 \mathrm{~b}$ \\
112,5 & $186,3 \pm 37,2 \mathrm{a}$ & $354,3 \pm 72,4 \mathrm{~b}$ \\
\hline
\end{tabular}

*Valores seguidos por letras distintas na mesma coluna diferem entre si pelo teste Tukey, a 5\% de significância.

Os tempos de latência e recuperação expostos anteriormente são semelhantes aos encontrados por Okamoto et al. (2009) com juvenis de Trachinotus marginatus. Os autores encontraram intervalos variando entre 2 e 11 minutos na indução e 3 a 14 minutos para a recuperação dos peixes, utilizando o mesmo produto. Todavia, no presente estudo, alguns desses valores foram superiores ao preconizado por Roubach e Gomes (2001) como tempos permitidos para os animais sofrerem a indução anestésica profunda, bem como para recuperação ao comportamento normal.

Em lambaris-do-rabo-amarelo (Astyanax altiparanae), Gimbo et al. (2008) testaram a eficiência do fármaco e relataram tempos inferiores aos encontrados no presente estudo. Entretanto, os pesquisadores observaram que, quando aplicadas concentrações de $125 \mathrm{mg} . \mathrm{L}^{-1}$, houve mortalidade dos animais. Contudo, 100mg. $\mathrm{L}^{-1}$ apresentaram os melhores resultados. No estudo com quinguio, esse anestésico não proporcionou mortalidade dos animais, sendo eficaz e seguro aos peixes.

Anziliero et al. (2008) também expõem tempos inferiores na indução e recuperação de alevinos de jundiá com $100 \mathrm{mg} . \mathrm{L}^{-1}$ do produto. Gomes et al. (2001) encontraram resultados de indução e perda de equilíbrio em menos de 2 minutos quando colocados em doses de 50 a $100 \mathrm{mg} . \mathrm{L}^{-1} \mathrm{e}$ tempos superiores a 7 minutos para a recuperação. Com concentrações acima de $150 \mathrm{mg} . \mathrm{L}^{-1}$, os animais rapidamente atingiram estado de dormência e longos tempos para o recobramento (acima de 10 minutos) dos padrões normais. 
Na literatura, a benzocaína foi testada em outras espécies de peixes, como carpas (Cyprinius carpio) (Antunes, et al., 2008), atlantic cod (Gadus morhua) (Zahl et al., 2009), atlantic salmon (Salmo salar) (Iversen et al., 2003), tilápia do Nilo (Oreochromis niloticus) (Gontijo et al., 2003), entre outros. Em todos os casos, os autores relatam eficiência em alguma das concentrações testadas, o que pode variar também conforme o tamanho dos peixes.

Segundo Inoue et al. (2004), a benzocaína causa menos efeito colateral na anestesia profunda de matrinxã, além de ser mais potente, necessitando de menor dosagem para a latência dos animais. Bastos-Ramos et al. (1998) concluem que acima de $100 \mathrm{mg} . \mathrm{L}^{-1}$ é eficiente para a sedação entre 6 e 8 minutos após a aplicação e a anestesia tem efeito até 15 e 20 minutos, causando pequena depressão respiratória.

Dentre as diversas opções de anestésicos disponíveis, a benzocaína mostrou-se eficiente na latência dos quinguios, pelo fato de o fármaco não ter causado mortalidade dos animais e estes terem recobrado o sentido normal posteriormente. Contudo, a disponibilidade do eugenol, mesmo não sendo recomendado pelo Conselho Federal de Medicina Veterinária como um anestésico seguro para a eutanásia de peixes, em anestesias para manejos é eficaz quando utilizado com precaução.
O eugenol é um composto fenólico volátil e é o principal constituinte do óleo de cravo (Mazzafera, 2003). Ele vem sendo utilizado como um fármaco alternativo na sedação de peixes (Roubach, 2005). Porém, já existem diversos estudos que podem fazer com que o produto passe de alternativo para convencional, inclusive com margens de segurança para as diferentes espécies de peixes.

No presente estudo, o eugenol mostrou ser um produto eficiente para latência dos animais (Tab. $3)$. Entretanto, os tempos foram mais elevados quando comparados à benzocaína. Houve diferenças significativas $(\mathrm{P}<0,05)$ entre as dosagens para os estágios de anestesia e recuperação. Tratamentos com doses inferiores a $37,5 \mathrm{mg} . \mathrm{L}^{-1}$ não foram suficientes para induzir o peixe à sedação. Contudo, valores de $75 \mathrm{mg} . \mathrm{L}^{-1}$ acarretaram em morte de $25 \%$ dos animais, além de apresentar tempo de recuperação acima de 15 minutos, não sendo recomendado segundo Roubach e Gomes (2001).

A dose com melhor índice de tempo para os dois estágios foi a de $50 \mathrm{mg} . \mathrm{L}^{-1}$ que, além de não proporcionar morte aos animais, não diferiu estatisticamente do tempo apresentado pela concentração de $62,5 \mathrm{mg} . \mathrm{L}^{-1}$.

Tabela 3. Tempo (em segundos) de indução e recuperação dos quinguios expostos a diferentes concentrações de eugenol

\begin{tabular}{lcc}
\hline Tratamento* & Anestesiado & Recuperado \\
\hline 37,5 & $535,6 \pm 42,6 \mathrm{~b}$ & $489,0 \pm 190,0 \mathrm{a}$ \\
50,0 & $395,1 \pm 60,3 \mathrm{a}$ & $579,0 \pm 113,6 \mathrm{ab}$ \\
62,5 & $316,6 \pm 113,1 \mathrm{a}$ & $806,6 \pm 148,8 \mathrm{bc}$ \\
75,0 & $293,1 \pm 50,0 \mathrm{a}$ & $912,5 \pm 257,7 \mathrm{c}$ \\
\hline
\end{tabular}

*Valores seguidos por letras distintas na mesma coluna diferem entre si pelo teste Tukey, com 5\% de significância.

O tempo de recuperação foi dependente da concentração, ou seja, quanto menor a dose maior o tempo para anestesia e menor para recuperação. Inoue et al. (2003) observaram independência para o tempo de recuperação de juvenis de matrinxã Brycon cephalus e encontraram tempos inferiores para os dois estágios. Vidal et al. (2007) também encontraram tempos inferiores; juvenis de matrinxã com peso médio de $3,3 \mathrm{~g}$ demoraram apenas 3 minutos para se recuperar na dose de $50 \mathrm{mg} . \mathrm{L}^{-1}$, enquanto no presente estudo ultrapassaram 9 minutos.

Gonçalves et al. (2008), ao pesquisarem anestésicos em pacu, relatam que a dose de $50 \mathrm{mg} . \mathrm{L}^{-1}$ de eugenol se mostra eficiente na sedação dos animais. Contudo, os valores obtidos foram inferiores ao do presente trabalho, em que, em menos de um minuto, houve a anestesia profunda, e, em pouco mais de dois minutos, os animais já estavam recuperados. Hisano et al. 
(2008), ao estudarem juvenis de dourado (Salminus brasiliensis) com peso médio de 20 gramas, relatam que os tempos de indução variaram entre 1,5 e 11 minutos com as doses de 60 e $20 \mathrm{mg}^{-\mathrm{L}^{-1}}$ de eugenol, respectivamente. Embora considerados tempos longos, os valores são inferiores aos observados na indução do quinguio. Isso pode sugerir que, quanto menor é o tamanho do peixe, maior é o tempo gasto na latência dos animais. Contudo, cabe ressaltar que os níveis diferenciam-se de espécie para espécie, principalmente porque o quinguio não é um peixe agitado. Esse fato pode colaborar para os tempos do anestésico serem elevados em relação aos peixes mais agitados, como os dourados e os matrinxãs.

Em pesquisa com o pintado (Pseudoplatystoma corruscans), Vidal et al. (2006) observaram tempos de indução inferiores a um minuto com concentrações de 50, 75 e 100mg. $\mathrm{L}^{-1}$ de eugenol, Porém, na maior concentração do anestésico, houve a morte de um animal. Isso sugere que, em doses elevadas, essa substância pode ser tóxica aos peixes, impossibilitando que estes retomem as características propícias para o cultivo.

Os resultados descritos por Vidal et al. (2008) com tilápia-do-nilo mostraram ser baixos em relação aos tempos dos quinguios. As tilápias atingiram a sedação e recuperação em pouco mais de dois minutos. Juvenis de senegalese sole (Solea senegalensis), expostos a $40 \mathrm{mg} . \mathrm{L}^{-1} \mathrm{de}$ eugenol, entraram em estágio IV de anestesia com menos de 2 minutos e se recuperaram em menos de 4 minutos (Weber et al., 2009).

Juvenis de tambaqui (Colossoma macropomum), submetidos a diferentes concentrações do anestésico eugenol, foram estudados por Roubach et al. (2005), que obtiveram tempo de 3,5 minutos para indução e 6,5 minutos para recuperação. Todas essas variações nos tempos e concentrações nos permitem inferir na disponibilidade desse produto como um fármaco para a utilização nos manejos de anestesia em peixes. Como reportado acima, os tempos podem ser superiores ou inferiores, de acordo com a espécie em que se está trabalhando, assim como se o peixe é de couro ou escama, e outra questão que deve ser levada em conta é a idade ou fase de vida, que pode necessitar de mais ou menos concentração conforme o metabolismo do animal.
Todos esses resultados reforçam a ideia de que cada espécie de peixe tem um tempo específico para a indução anestésica e, conforme a sua fisiologia, esse tempo pode perdurar para a volta da sensação e estímulo dos animais. Além das diferenças entre as espécies, outro fator que pode influenciar uma sedação é o estágio de vida em que o animal se encontra. Como existe necessidade de manejos em todas as etapas de desenvolvimento, reprodução, transporte e cirurgias, torna-se necessário estudar a eficiência dos diferentes tipos de produtos anestesiantes para que haja menos estresse nos peixes e não ocorra eventual mortalidade nas práticas rotineiras aplicadas aos animais.

\section{CONCLUSÕES}

Os dois diferentes fármacos mostraram ser eficientes na latência anestésica dos animais. A dose resposta mais eficiente para a indução e recuperação anestésica de benzocaína foi de $87,5 \mathrm{mg} . \mathrm{L}^{-1}$, enquanto a melhor dose de eugenol foi de $50 \mathrm{mg} \cdot \mathrm{L}^{-1}$.

\section{REFERÊNCIAS}

ANTUNES, M.I.P.P.; SPURIO, R.S.; GODOI, D.A. et al. Cloridrato de benzocaína na anestesia de carpas (Cyprinius carpio). Semina Cienc. Agr., v.29, p.151156, 2008.

ANZILIERO, D.; KREUTZ, C.L.; BARCELLOS, G.J.L. Avaliação de quatro diferentes anestésicos para sua utilização no manejo de jundiás (Rhamdia quelen). In: CONGRESSO BRASILEIRO DE MEDICINA VETERINÁRIA, 35., 2008. Gramado, RS. Anais... Gramado, 2008.

BANDYOPADHYAY, P.; SWAIN, S.K.; MISHRA, S. Growth and dietary utilization in goldfish (Carassius auratus Linn.) fed diets formulated with various local agro-produces. Biores. Techon., v.96, p.731-740, 2005.

BASTOS-RAMOS, W.P.; GONÇALVES, N.M.F.M.; BACILA, M. Anesthesia and analgesia in antartic fish: na experimental approach. Arch. Vet. Sci., v.3, p.95100, 1998.

CONSELHO Federal de Medicina Veterinária, Resolução 714 de 20 de junho de 2002. Procedimentos e métodos de eutanásia em animais. Brasil, 2002.

GIMBO, R.Y.; SAITA, M.V.; GONÇALVES, A.F.N.; TAKAHASHI, L.S. Diferentes concentrações de benzocaína na indução anestésica do lambari-do-raboamarelo (Astyanax altiparanae). Rev. Bras. Saúde. Prod. Anim., v.9, p.350-357, 2008. 
GOLOMBIESKI, J.I.; MARCHEZAN, E.; MONTI, M.B. et al. Qualidade da água no consórcio de peixes com arroz irrigado. Ciênc. Rural, v.35, p.1263-1268, 2005.

GOMES, L.C.; CHIPPARI-GOMES, A.C.; LOPES, N.P. et al. Efficacy of benzocaine as an anesthetic in juvenile tambaqui Colossoma macropomum. J. W. Aquac. Soc., v.32, p.426-431, 2001.

GONÇALVES, A.F.N.; SANTOS, E.C.C.; FERNANDES, J.B.K.; TAKAHASHI, L.S. Mentol e eugenol como substitutos da benzocaína na indução anestésica de juvenis de pacu. Acta Sci. Anim. Sci., v.30, p.339-344, 2008.

GONTIJO, A.M.M.C.; BARRETO, R.E.; SPEIT, G. et al. Anesthesia of fish with benzocaine does not interfere with comet assay results. Mut. Res., v.534, p.165-172, 2003.

HISANO, H.; ISHIKAWA, M.M.; FERREIRA, R.A. et al. Tempo de indução e de recuperação de dourado Salminus brasiliensis (Cuvier, 1816), submetidos a diferentes concentrações de óleo de cravo Eugenia sp. Acta Sci. Anim. Sci., v.30, p.303-307, 2008.

HONCZARYK, A.; INOUE, L.A.K.A. Anestesia do pirarucu por aspersão direta nas brânquias do eugenol em solução aquosa. Cienc. Rural, v.39, p.577-579, 2009.

INOUE, L.A.K.A.; SANTOS NETO, C.; MORAES, G. Benzocaína como anestésico para juvenis de matrinxã (Brycon cephalus). Bol. Téc. CEPTA, v.15. p.23-30, 2002.

INOUE, L.A.K.A.; SANTOS NETO, C.; MORAES, G. Clove oil as anaesthetic for juveniles of matrinxã Brycon cephalus (Gunther, 1869). Cienc. Rural, v.33, p.943-947, 2003.

INOUE, L.A.K.A.; HACKBARTH, A.; MORAES, G. Avaliação dos anestésicos 2-phenoxyethanol e benzocaína no manejo do matrinxã Brycon cephalus (Günther, 1869). Biodiversidade Pampeana, v.2. p.1015, 2004.

IVERSEN, M.; FINSTAD, B.; McKINLEY, R.S.; ELIASSEN, R.A. The efficacy of metomidate, clove oil, aqui-s and benzoak as anesthetics in atlantic salmon (Salmo salar L.) smolts, and their potential stress-reducing capacity. Aquaculture, v.221, p.549566, 2003.
MAZZAFERA, P. Efeito alelopático do extrato alcoólico do cravo-da-índia e eugenol. Rev. Bras. Botânica, v.26, p.231-238, 2003.

OKAMOTO, M.H.; TESSER, M.B.; LOUZADA, L.R. et al. Benzocaína e eugenol como anestésicos para juvenis do pampo (Trachinotus marginatus). Cienc. Rural, v.39, p.866-870, 2009.

ROUBACH, R.; GOMES, L.V. O uso de Anestésico durante o manejo de peixes. Panorama $d a$ Aquicultura, v.11, p.37-40, 2001.

ROUBACH, R.; GOMES, L.C.; FONSECA, F.A.L.; VAL, A.L. Eugenol as an efficacious anaesthetic for tambaqui, Colossoma macropomum (Couvier). Aquaculture Research, v.36, p.1056-1061, 2005.

SAS Institute Inc. SAS User's guide statistics. 9.ed, Cary, North Caroline: SAS Institute Inc., 9.1.3. 2004.

SOARES, C.M.; HAYASHI, C.; GONÇALVES, G.S. et al. Plâncton, Artemia sp, dietas artificiais e suas combinações no desenvolvimento e sobrevivência do quinguio (Carassius auratus) durante a larvicultura. Acta Sci., v.22, p.383-388, 2000.

VIDAL, L.V.O.; ALBINATI, R.C.B.; ALBINATI, A.C.L.; MACÊDO, G.R. Utilização do eugenol como anestésico para o manejo de juvenis de pintado (Pseudoplatystoma corruscans). Acta Sci. Biol. Sci., v.28, p.275-279, 2006.

VIDAL, L.V.O.; FURUYA, W.M.; GRACIANO, T.S. et al. Eugenol como anestésico para juvenis de matrinxã (Brycon cephalus). Rev. Bras. Saúde Prod. Na., v.8, p.335-342, 2007.

VIDAL, L.V.O.; ALBINATI, R.C.B.; ALBINATI, A.C.L. et al. Eugenol como anestésico para tilápia do Nilo. Rev. Agropec. Bras., v.43, p.1069-1074, 2008.

ZAHL, I.H.; KIESSLING, A.; SAMUELSEN, O.B.; HANSEN, M.K. Anaesthesia of Atlantic cod (Gadus morua) - effect of pre anaesthetic sedation, and importance of body weight, temperature and stress. Aquaculture, v.295, p.52-59, 2009.

WEBER, R.A.; PELETEIRO, J.B.; GARCIA MARTIN, L.O.; ALDEGUNDE, M. The efficacy of 2-phenoxyethanol, metomidate, clove oil and MS-222 as anaesthetic agents in the Senegalese sole (Solea senegalensis Kaup 1858). Aquaculture, v.288. p.147150, 2009.

WOODY, C.A.; NELSON, J.; RAMSTAD, K. Clove oil as an anaesthetic for adult sockeye salmon: field trials. J. Fish Biol., v.60. p.340-347, 2002. 